\title{
Investigating Cytoskeletal Alterations as a Potential Marker of Retinal and Lens Drug-Related Toxicity
}

\author{
Maria E. Verdugo-Gazdik, ${ }^{1}$ Damir Simic, ${ }^{1}$ Alan C. Opsahl, ${ }^{1}$ and Mark W. Tengowski ${ }^{2}$
}

\begin{abstract}
Actin filaments play a critical role in the normal physiology of lenticular and retinal cells in the eye. Disruption of the actin cytoskeleton has been associated with retinal pathology and lens cataract formation. Ocular toxicity is an infrequent observation in drug safety studies, yet its impact to the drug development process is significant. Recognizing compounds through screening with a potential ocular safety liability is one way to prioritize development candidates while reducing development attrition. Lens epithelial cells from human, dog, and rat origins and retinal pigmented epithelium cells from human, monkey, and rat origins were cultured and investigated with immunocytochemical techniques. Cells were treated using noncytotoxic doses of the compound, fixed, stained for actin with rhodamine phalloidin, and counterstained for nuclei with TOTO-3, followed by confocal imaging. Tamoxifen and several experimental compounds known to be in vivo lens and retinal toxicants caused a reduction in F-actin fluorescence at noncytotoxic concentrations in all cells tested as observed by confocal microscopy. Developing an assay that predicts ocular toxicity helps the development process by prioritizing compounds for further investigation. Drug-induced cytoskeletal alterations may be useful as a potential safety-screening marker of retinal and lens toxicity. The knowledge of actin molecular biology and the application of other mechanistic screens to toxicology are discussed. Reducing this work to a high-throughput platform will enable chemists to select compounds with a reduced risk of ocular toxicity.
\end{abstract}

\section{Introduction}

A CTIN CYTOSKELETON is essential in maintaining ocular cell symmetry and architecture-ultimately preserving lens transparency and refractive power-as it plays a key role in the correct physiological function of the lens and retinal pigmented epithelium. Although recent studies have demonstrated the effects of drug toxicity on a group of proteins known to regulate the organization of the actin cytoskeleton, little is known about the effects of ophthalmologic-toxic agents on actin itself. ${ }^{1-3}$ Observations drawn from studies in cell biology as well as those documenting the effects of pharmaco- logic agents on the eye suggest that changes in the structure of actin filaments could serve as a potential marker of drug-induced retinal and lens toxicity. ${ }^{3-10}$

Both the retinal pigmented epithelium and lens epithelium carry out several functions that are crucial for the normal function of the eye. Located between the photoreceptors and the choiroid, retinal pigmented epithelial cells function mainly to support the overlying photoreceptor cells. They carry out this support function by transporting nutrients, storing vitamin A, absorbing stray light not absorbed by the photoreceptors, and - perhaps most critically - undertaking the "scavenging" role of phagocytosis of the rod outer segments. ${ }^{11-14}$ Lens epithelial

Pfizer Global Research \& Development, ${ }^{1}$ Groton-New London Laboratories, Groton, CT; and ${ }^{2}$ Michigan Laboratories, Ann Arbor, MI.

ABBREVIATIONS: ADF, actin depolymerizing factor; CAT, catalytic domain; DMEM, Dulbecco's Modified Eagle's Medium; FBS, fetal bovine serum; GTPase, guanosine triphosphatase; HMG-CoA, 3-hydroxy-3-methylglutaryl coenzyme A; LAT-A, latrunculin-A; LPA, lysophosphatidic acid; NA, numerical aperture; PBS, phosphate-buffered saline. 
cells are located under the anterior lens capsule. They migrate, proliferate, and differentiate into lenticular fiber cells at the equatorial bow of the lens. ${ }^{4}$ As these cells become part of the cortex, they elongate and begin losing organelles such as nuclei and mitochondria. The actin cytoskeleton plays several important roles in the functioning of the lens and retinal pigmented epithelium. Actin is associated with adherent junctions between fiber and epithelial cells, and it contributes to the migration and elongation of lens cells. ${ }^{4,5}$ During tension and the process of accommodation that occur in the eye, actin filaments form polygonal arrays that stabilize the lens. With respect to retinal pigmented epithelial cells, actin microfilaments are essential for correct internalization and phagocytosis of outer segments, and the microfilaments are a key component of microvilli protrusions that surround rod outer segments. ${ }^{15-17}$

Understanding the biochemical and molecular mechanisms that control the organization of actin is therefore a major goal of contemporary cell biology, with implications for health and disease. On a molecular level, actin is regulated directly via the Rho family of small G proteins, including Rho, Rac, and Cdc42. ${ }^{6,18,19}$ In addition, extensive crosstalk takes place between guanosine triphosphatases (GTPases) of the Ras and Rho subfamilies: both Ras and Cdc42 can activate Rac, while Rac in turn can activate Rho. Cdc42, Rac, and Rho proteins also play a cooperative role in the protein-induced transformation of the Ras subfamily. ${ }^{20-22}$ Based on such observations, researchers such as Hall ${ }^{6}$ have suggested that Rho GTPases are key regulatory molecules linking surface receptors to the organization of the actin cytoskeleton. Consequently, many factors can affect actin assembly by influencing the Rho family of proteins.

In recent years, studies have linked Rho-regulated actin cytoskeleton organization with corneal epithelial migration, with implications for corneal disease. ${ }^{1,2}$ It is suggested that cholesterol-lowering drugs such as lovastatin impair Rho and Rac GTPase function, leading to cytoskeletal changes in lens epithelial cells. The implications for cataract formation are significant. Pretreatment of lens epithelial cells with such agents as the RhoGTPase inhibitor C3-exoenzyme interferes with the ability of certain growth factors to trigger activation of Rho and Rac GTPases and, by extension, actin stress fiber and focal adhesion formation. ${ }^{3}$

Research has been published in recent years on actin cytoskeleton from a microbiological perspective. . $^{73,24}$ Although enough is known about the effect of certain potent pharmaceutical agents on the eye to raise clinical concerns, much less is known about the effect of drug toxicity at the molecular level..$^{25-27}$ It is thought that changes in the structure of actin microfilaments potentially may reflect injury to lens and retinal pigmented epithelial cells consistent with the effects of drug toxicity, but this has not been widely demonstrated. ${ }^{5,28-30}$ What is clear is that toxicity in the eye can be caused by a wide array of medications, resulting from heterogeneous forms of pathogenesis and leading to a diverse set of adverse effects. ${ }^{25}$ Sometimes the effects are directly related to drug exposure itself; at other times they can be caused by secondary effects of the drug (e.g., metabolic product/biotransformation to reactive intermediate). Some of the observed side effects are reversible; others are not. For example, tamoxifen, an oral anti-estrogen used in the treatment of breast cancer, has been associated with such eye problems as cataracts and retinopathy. ${ }^{26,31,32}$ Furthermore, tamoxifen has been shown to inhibit phagocytosis of rod outer segments, ${ }^{14}$ an important function of retinal pigmented epithelial cells and a function for which action microfilaments are essential. If the cytotoxicity of a drug such as tamoxifen could be shown to affect the cytoskeleton early, before cytotoxicity has been known to occur using such standard assays as the WST-1 dye test, actin could potentially serve as a marker of drug-induced lens and retinal changes preceding loss of cell viability.

Early in vivo toxicology assessment of drug development candidates traditionally focuses on the "big" organs, such as the liver, kidneys, heart, brain, lung, and testis, since these are major causes of toxicity-related development attrition. Ocular toxicity, on the other hand, is not really evaluated until much further in development and, when identified, can be much more costly to a program, since this is a significant finding that tends to be a development stop. Industrial toxicology over the past decade has switched from risk identification to risk management. This is fine for liver toxicity where serum chemistries can be correlated to potential liver injury. In cases of ocular toxicity, there are few risk management tools, and the acceptability of these safety risks is very low. With this knowledge in mind, efforts to reduce latestage attrition by identifying toxicity screening assays have increased in the area of toxicology. When there are thousands of drug candidates to evaluate, and it is known that a certain pharmacology or chemical structure is predisposed to cataract formation, any indication or ability to prioritize compounds is warranted. In our investigation into developing a screen for ocular toxicity, we had the benefit of knowing the in vivo result and are working to reverse-engineer an in vitro screen that could be a predictive marker of imminent lens toxicity.

This research effort grew out of a set of cell culture experiments evaluating different preparation methods for evaluating the cytoskeleton. In a parallel set of investigations, we were brainstorming opportunities to screen away from potential ocular toxicity through the development of a cell viability assay. Many cell lines were at our disposal. Why actin and not microtubules became the eventual focus of the effort centered on an observation 
associated with the cell viability screening, namely, that with increasing concentration of drugs with known in vivo cataract liability, a concomitant in vitro cell death also occurred. And when these cells died, they lifted from the plate. Since cell adhesion in culture tends to be mediated by actin, we looked for changes in actin preceding loss of adhesion as a possible marker of cellular toxicity. Actin staining was also observed not to change in culture when using compounds of similar pharmacology but without cataracts, hence the possibility of actin change to screen for cataract potential. By evaluating the organization of the F-actin cytoskeleton using confocal microscopy, quantifying the fluorescence of F-actin, and assessing cell viability over the 24-h treatment period, this study sought to investigate the extent of structural modification in actin filaments seen in cells exposed to increasing concentrations of tamoxifen before evident cytotoxicity was present. We are continuing to investigate the sensitivity and specificity of this assay to predict or translate into a cataract liability. Utilizing this screen, even without this quantitative information, does achieve the goal of helping prioritize development candidates, as these potential failures could be tested by collecting the eyes early than normal in the discovery process.

\section{Materials and Methods}

In this study, lens epithelial cells (human and rat) and retinal pigmented epithelial cells (human, monkey, and rat) were grown to $80 \%$ confluency, treated for $24 \mathrm{~h}$ with increasing concentrations of tamoxifen, and compared to untreated controls using a variety of measures as explained in the following sections.

\section{Primary cell culture}

Sprague-Dawley rats and primates were obtained from Charles River (Wilmington, MA); eye collection was carried out in accordance with guidelines of the Association for Research in Vision and Ophthalmology for animal care and use. Following enucleation, monkey and rat eyes were opened by an incision at the ora serrata, followed by a circumferential incision to separate the posterior segment of the eye from the anterior segment, in order to harvest the retina. For monkey retinal pigmented epithelial cell lines, the posterior segment was placed in an eye holder and washed with Puck's saline F solution (containing the following $\mathrm{wt} / \mathrm{vol}$ concentrations in water: $0.74 \% \mathrm{NaCl}, 0.018 \%$ $\mathrm{Na}_{2} \mathrm{HPO}_{4}, \quad 0.0083 \% \quad \mathrm{KH}_{2} \mathrm{PO}_{4}, \quad 0.03 \% \mathrm{KCl}, \quad 0.12 \%$ $\mathrm{NaHCO}_{3}, 0.11 \%$ glucose, and $0.0034 \%$ EDTA) at $\mathrm{pH}$ 7.12. The retinal layer was gently removed. Once clean, this tissue was incubated for $40 \mathrm{~min}$ in $0.25 \%$ trypsin at $37^{\circ} \mathrm{C}$. Trypsin medium was collected and placed in a 15-ml Falcon tube together with $10 \mathrm{ml}$ of Dulbecco's Modified Eagle's Medium (DMEM) $\left(\right.$ GIBCO $^{\circledR}$, Grand Island, NY) containing $15 \%$ fetal bovine serum (FBS) (Hyclone, Logan, UT) and spun at $500 \mathrm{rpm}$ for $5 \mathrm{~min}$. This incubation/collection with trypsin and DMEM containing 5\% FBS was repeated for at least seven times, removing the supernatant and concentrating the cells into a soft pellet. The final cell pellet was added to DMEM containing $15 \%$ FBS to a cell culture flask. Cells were then plated at a density of $4 \times 10^{4} \mathrm{cells} / \mathrm{cm}^{2}$ cells in a T25 flask with DMEM containing 15\% FBS, $1 \%$ L-glutamine (GIBCO), and 1\% penicillin-streptomycin (GIBCO). Cells were maintained for 2 weeks in $95 \%$ air and $5 \% \mathrm{CO}_{2}$ at $37^{\circ} \mathrm{C}$ under $100 \%$ humidity. RPE-J is a commercially available retinal pigmented epithelial cell line derived from primary cultures of retinal pigmented epithelial cells taken from 7-dayold Long-Evans rats (http://www.atcc.org/common/ catalog/numSearch/numResults.cfm? atccNum $=$ CRL2240).

Rat primary lens epithelial cells were isolated by separating the capsule from the lens nucleus. The lens capsule was added to unsupplemented collagenase medium $(1 \mathrm{mg} / \mathrm{ml})$, incubated at $37^{\circ} \mathrm{C}$, and monitored every $3-5$ min for dissociation. Cells were then plated on dishes coated with collagen IV at a density of $1 \times 10^{4} \mathrm{cells} / \mathrm{cm}^{2}$ (Biocoat Cellware, collagen type IV, VWR 62405, Becton Dickinson, San Jose, CA). Growth medium consisting of DMEM low glucose (GIBCO), $15 \%$ FBS, $1 \%$ penicillin-streptomycin, $1 \%$ L-glutamine, and $25 \mathrm{~m} M$ HEPES was added. Cells were grown to confluence before being trypsinized and plated for experiments.

\section{Cell line culture}

The rat retinal pigmented epitheial cell line RPE-J was grown in DMEM containing 4\% FBS, $0.1 \mathrm{mM}$ nonessential amino acids, and $4.5 \mathrm{~g} / \mathrm{L}$ glucose. Cells from a human RPE cell line (ARPE-19) were treated with DMEM/F-12 medium (GIBCO) containing 10\% FBS and $2 \mathrm{mM}$ L-glutamine and maintained in $95 \%$ air and $5 \%$ $\mathrm{CO}_{2}$ at $37^{\circ} \mathrm{C}$ under $100 \%$ humidity. A human lens epithelial cell line (hLEC SRA 04/01) was established by transforming primary lens cells with a large T-antigen of SV $40 .{ }^{33}$ Cells were grown in DMEM containing $15 \%$ FBS in a mixture of $95 \%$ air and $5 \% \mathrm{CO}_{2}$ at $37^{\circ} \mathrm{C}$ under $100 \%$ humidity.

\section{Cell plating and treatments}

For the F-actin assay, all cells were plated in fourchamber, polystyrene, tissue-culture-treated glass slides (Falcon BD, San Jose) at a density of $5 \times 10^{4}$ cells per chamber. Once the cells reached $80 \%$ confluency, they were treated with increasing concentrations of tamoxifen 
(tamoxifen citrate, Calbiochem, San Diego, CA). Cells were grown to $80 \%$ confluency and treated with increasing concentrations of tamoxifen. With a mixture of cell lines and primary cell cultures in this investigation and using cell viability assays as a guide, it should be noted that each cell line responds differently to tamoxifen exposure. Given that some cultures are more robust or react differently to the stresses of culture in this screening study, tamoxifen concentrations were optimized per culture to create exposures of a nontoxic, upper limit of viability, and cytotoxic levels.

\section{F-actin staining and confocal microscopy}

Cultured cells were rinsed twice in phosphatebuffered saline (PBS) (Sigma-Aldrich, St. Louis, MO), fixed with 4\% paraformaldehyde (Electron Microscopy Sciences, Hatfield, PA) at room temperature for $30 \mathrm{~min}$, and rinsed again in PBS. To quench any remaining paraformaldehyde, cells were incubated for $10 \mathrm{~min}$ in $100 \mathrm{~m} M$ PBS/glycine (Sigma-Aldrich). Fixed cells were permeabilized for $20 \mathrm{~min}$ at room temperature with $0.1 \%$ Triton X-100 (Sigma-Aldrich) in PBS. Cells were incubated for $1 \mathrm{~h}$ in a solution containing $1 \%$ bovine serum albumin (Roche, Indianapolis, IN), 1 $\mathrm{mg} / \mathrm{ml}$ RNase A (Sigma-Aldrich), and PBS. Stock of rhodamine phalloidin (Molecular Probes, Eugene, OR), which was made by adding $1.5 \mathrm{ml}$ of methanol to 300 $\mathrm{U}$ of solid stock, was diluted in blocking buffer by a ratio of 1:400, resulting in a final concentration of 100 $\mathrm{n} M$. F-actin and nuclei were detected by incubating cells for $1 \mathrm{~h}$ with rhodamine phalloidin and $1 \mu \mathrm{M}$ TOTO-3 (Molecular Probes) in PBS. Following staining, cells were washed three times in PBS at room temperature, each time for $5 \mathrm{~min}$. Coverslips (24 ( $50 \mathrm{~mm}$, VWR, West Chester, PA) were mounted onto a slide face down, with the use of a mounting anti-fade solution (Molecular Probes). After $24 \mathrm{~h}$ of incubation in the dark, fingernail polish was used to seal the slide. Confocal microscopy was performed with a Leica SP laserscanning microscope (Leica, Heidelberg, Germany) using an objective of $40 \times(1.25$ numerical aperture [NA] $)$ with oil.

\section{Cell viability}

Cells were cultured in 96-well plates (Costar, Corning, NY) at $1 \times 10^{4}$ cells per well and grown to $80 \%$ confluency. After being treated for $24 \mathrm{~h}$ with increasing concentrations of tamoxifen, cells were washed twice in PBS using a soaking pipette (VWR, West Chester, PA), and WST-1 reagent was added to the cells for $3 \mathrm{~h}$ at a ratio of $100 \mu \mathrm{l} / \mathrm{ml}$ of cell medium. The wells were analyzed by an enzyme-linked immunosorbent assay plate reader (Spectra Max Plus 384, Molecular Devices, Sunnyvale, CA) at a wavelength of $440 \mathrm{~nm}$.

\section{Results}

By treating a series of human and animal lens and retinal cells to increasing concentrations of tamoxifen for 24 $\mathrm{h}$ and comparing the outcomes to those in untreated controls, we were able to detect significant structural changes in (or affecting) the cells' actin filaments. For each group of cells, qualitative presentations of actin cytoskeleton using confocal microscopy demonstrating fluctuation in F-actin fluorescence and cell viability are provided. With the rat RPE-J cells, treated with concentrations of tamoxifen of $2.5 \mu M, 10 \mu M$, and $50 \mu M$, there was a detectible reduction in cell viability only at the highest concentration, while decreases in F-actin fluorescence were quantifiable beginning with the lowest concentration (Fig. 1). At $2.5 \mu M$ actin concentration was $80 \%$ of the concentration of untreated controls, and at $10 \mu \mathrm{M}$ it was still more than $50 \%$. However, at $50 \mu M$, actin concentration dropped to $<40 \%$. The qualitative images derived from confocal microscopy show that, as the concentration of tamoxifen increases, an increasing number of actin fibers are lost, suggesting a concentration-dependent relationship.

We assessed the effect of tamoxifen on human ARPE19 cells, treated with concentrations of the drug of 1 $\mu M, 12.5 \mu M$, and $25 \mu M$ (Fig. 2). The quantification of F-actin indicated that there was a loss of fluorescence

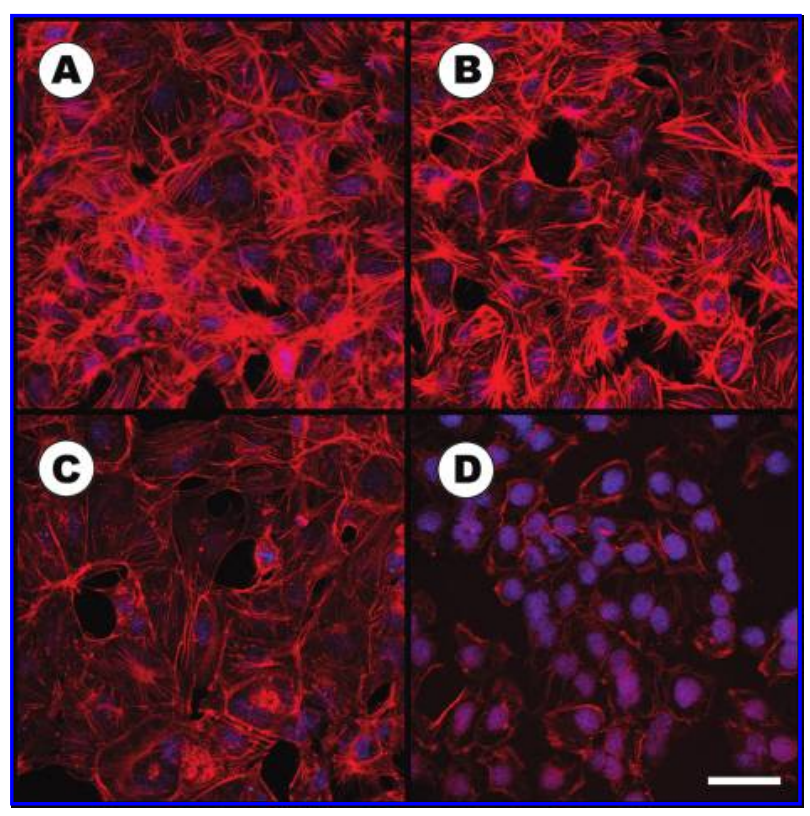

FIG. 1. Rat retinal pigmented epithelial cell line (RPE-3) was treated for $24 \mathrm{~h}$ with increasing concentrations of tamoxifen (A, $0 \mu \mathrm{M} ; \mathbf{B}, 2.5 \mu M ; \mathbf{C}, 10 \mu M$; and $\mathbf{D}, 50 \mu M$ ). F-actin cytoskeleton was stained with rhodamine phalloidin (red), and nuclei were stained with TOTO-3 dye (blue). Qualitative presentation of actin cytoskeleton using confocal microscopy ( $40 \times$ objective, 1.25 NA) shows a concentration-dependent loss of actin fibers with increasing concentration of tamoxifen. 


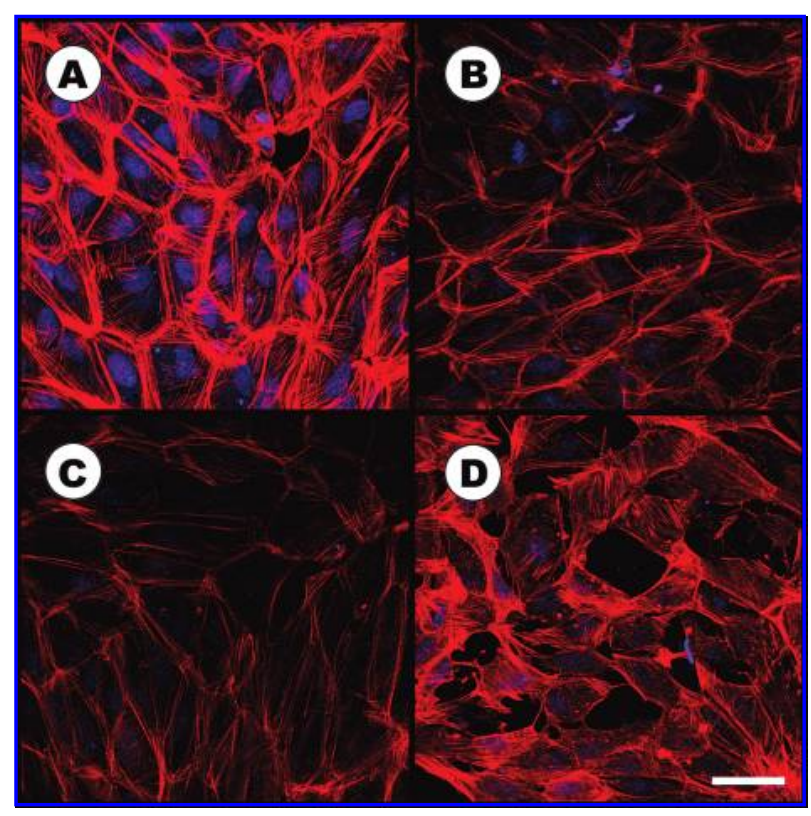

FIG. 2. Human retinal pigmented epithelial cell line (ARPE19) was treated for $24 \mathrm{~h}$ with increasing concentrations of tamoxifen (A, $0 \mu M$; B, $1 \mu M$; C, $12.5 \mu M$; and D, $25 \mu M$ ). Qualitative presentation of actin cytoskeleton using confocal microscopy ( $40 \times$ objective, $1.25 \mathrm{NA}$ ) shows a loss of F-actin at low noncytotoxic concentrations of tamoxifen $(1 \mu M)$, leading to eventual clumping at higher concentrations $(25 \mu M)$.

(to $<50 \%$ of that of controls) at low concentrations of tamoxifen $(1 \mu M)$. This loss is also reflected in the qualitative presentation, in which can be seen the decrease in F-actin at the lower concentrations of tamoxifen (1 $\mu M$ and $12.5 \mu M$ ) leading to eventual clumping at the highest concentration at which the ARPE-19 cells were tested $(25 \mu M)$. This aggregation of $\mathrm{F}$-actin, along with the loss of actin stress fibers, may provide the explanation for why a slight increase in fluorescence was seen at $25 \mu M$ tamoxifen (where it was $60 \%$ of that of controls, more than twice the F-actin concentration at 12.5 $\mu M)$. The third and final sets of retinal cells that we investigated were monkey primary retinal pigmented epithelial cells, and these were also treated with concentrations of tamoxifen of $1 \mu M, 12.5 \mu M$, and $25 \mu M$. Loss of F-actin fluorescence paralleled drops in cell viability but only at higher concentrations of tamoxifen (Fig. 3). At the $1 \mu M$ treatment, the concentration of Factin fluorescence actually increased $20 \%$ to that seen in untreated controls, and it was at $100 \%$ with $12.5 \mu M$ treatment, where cell viability began to drop to just below $80 \%$ of controls. The qualitative presentations suggest that the loss of actin fibers began as early as $1 \mu M$ treatment, and mirrors the loss in F-actin fluorescence only at $25 \mu M$.

Our assessment of lenticular cells resulted in comparable findings overall, but with even more precipi- tous drops in F-actin fluorescence than we saw with retinal cells. In rat primary lens epithelial cells treated with concentrations of tamoxifen of $5 \mu M, 25 \mu M$, and $75 \mu M$, the amount of F-actin fluorescence fell from $120 \%$ of the amount of controls at $5 \mu M$ to approximately $1 \%$ at $25 \mu M$ (Fig. 4) using a median measurement of the entire red channel (actin) and count of the nuclei in the blue channel. It should be noted that this reduction occurred at concentrations of tamoxifen considered to be noncytotoxic, and it is mirrored by the notable loss of actin fibers between $5 \mu M$ and $25 \mu M$ treatment that is apparent in the confocal microscopy images. We also examined cells from a human lens epithelial (SRA) cell line treated with concentrations of tamoxifen of $5 \mu M, 25 \mu M$, and $50 \mu M$. As we saw with human retinal pigmented epithelial cells, there was a reduction in $\mathrm{F}$-actin fluorescence at low, noncytotoxic concentrations of the drug (Fig. 5). This is shown in the qualitative presentation to be a rapid drop (to $<40 \%$ of the amount of F-actin of untreated controls). At 25 $\mu M, \mathrm{~F}$-actin fluorescence increased to $>60 \%$ of that of controls. Tamoxifen has started to become cytotoxic, resulting in the aggregation of actin fibers. Cell viability began to drop rapidly at concentrations $>25 \mu M$, suggesting that these levels are progressively cytotoxic. At $50 \mu M$ tamoxifen, a total loss of microfilaments is observed via confocal microscopy.

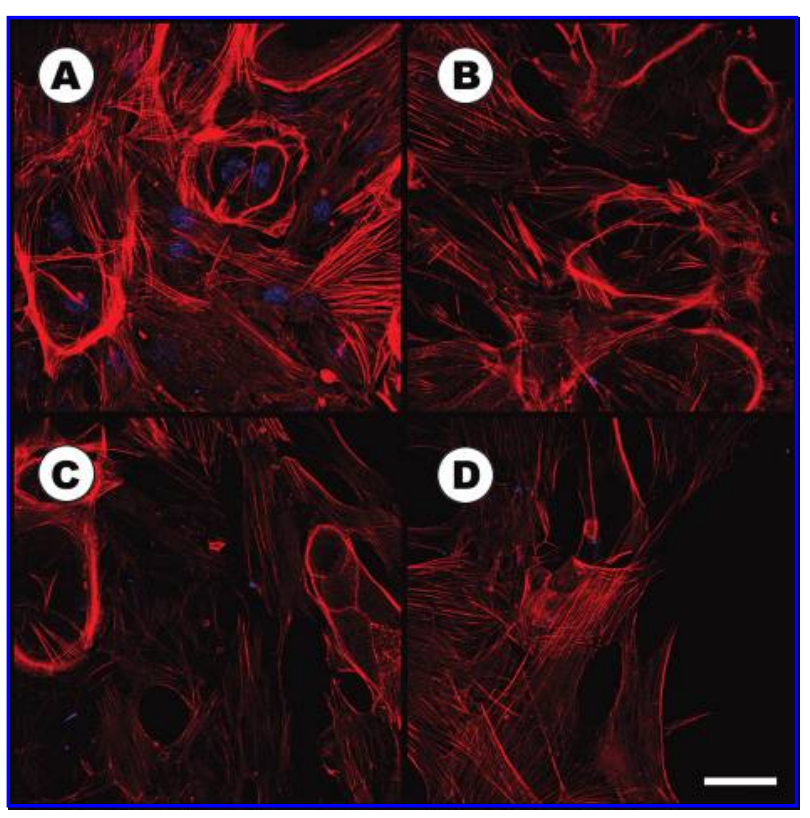

FIG. 3. Monkey primary retinal pigmented epithelial cells were treated for $24 \mathrm{~h}$ with increasing concentrations of tamoxifen $(\mathbf{A}, 0 \mu M$; $\mathbf{B}, 1 \mu M ; \mathbf{C}, 12.5 \mu M$; and $\mathbf{D}, 25 \mu M)$. Qualitative presentation of actin cytoskeleton using confocal microscopy ( $40 \times$ objective, $1.25 \mathrm{NA})$ shows a loss of actin fluorescence at higher concentrations of tamoxifen. 


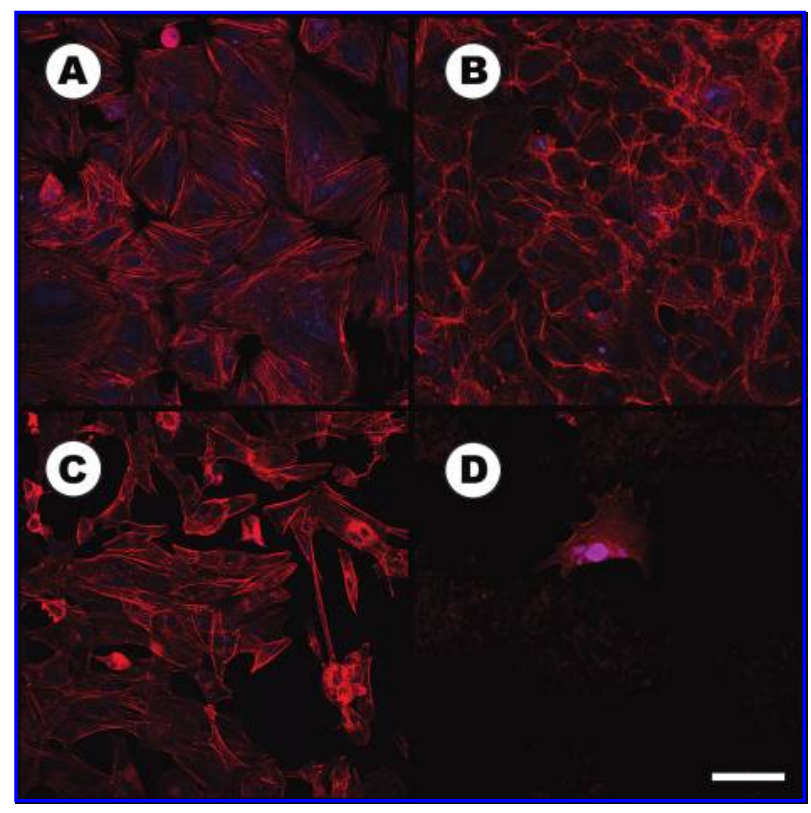

FIG. 4. Rat primary lens epithelial cells were treated for 24 $\mathrm{h}$ with increasing concentrations of tamoxifen $(\mathbf{A}, 0 \mu M ; \mathbf{B}, 5$ $\mu M ; \mathbf{C}, 25 \mu M$; and $\mathbf{D}, 75 \mu M$ ). Qualitative presentation of actin cytoskeleton using confocal microscopy $(40 \times$ objective, $1.25 \mathrm{NA}$ ) shows a loss of actin fluorescence at noncytotoxic concentrations of tamoxifen $(25 \mu M)$.

\section{Discussion}

In this study, we demonstrated that tamoxifen, known to be a lens and retinal toxicant in vivo, caused a reduction in F-actin fluorescence at noncytotoxic concentrations in all cell types tested. These findings suggest that drug-induced cytoskeletal alterations may be useful as an early marker of retinal and lens toxicity. If this new application of actin cytoskeleton can be qualified through the use of other drugs and compounds, it may point the way toward the development of an assay that could predict drug toxicity before any ocular damage becomes irreversible.

The rationale for why changes in actin cytoskeleton could serve as an early marker of toxicity to the eyes is based on a broad and diverse set of findings derived from studies in cell biology, ophthalmology, and toxicology. Furthermore, as much as is known about actin's direct role in cell growth, differentiation, morphogenesis, and motility, recent observations about the association of actin with other cellular proteins and growth factors have proven to be a comparably rich source for informing our working hypothesis about the effects of drug toxicity on the cytoskeleton.

Within the realm of cell biology, actin has been vividly described by McGough ${ }^{7}$ as "the molecular railroad track for myosin, the internal scaffold for microvilli, the molecular drill of the acrosomal reaction, and the comet on which pathogens travel." Discovered more than 50 years ago, purified from muscle, the protein is now recognized as the $42-\mathrm{kDa}$ actin monomer (G-actin), which assembles itself in vitro as well as in vivo to form long helical filaments known as F-actin. While much of our understanding of actin filaments comes from in vitro studies of the pure form of actin, the assembly and function of actin in the cell are governed by other proteins that bind actin. It is the interactions of these proteins ${ }^{34}$ that result in what McGough ${ }^{7}$ described as the "beautiful ballet" underlying cell differentiation and motility. "Disruptions in these processes," she wrote, "are the basis of many genetic diseases as well as of both viral and bacterial infectivity."

What becomes clear from a review of the literature detailing the many associations of actin cytoskeleton is that, even when it is not the central focus of a particular avenue of research, the protein remains at the center of processes within and affecting the cell. These associations include, but are not limited to, those involving microtubule dynamics and tubulin-interacting proteins, ${ }^{35,36}$ membrane trafficking and organelle transport, ${ }^{37}$ the WASP family of proteins and the Arp $2 / 3$ complex, ${ }^{38}$ gelsolin, villin, tropomodulin, and actin depolymerizing fac-

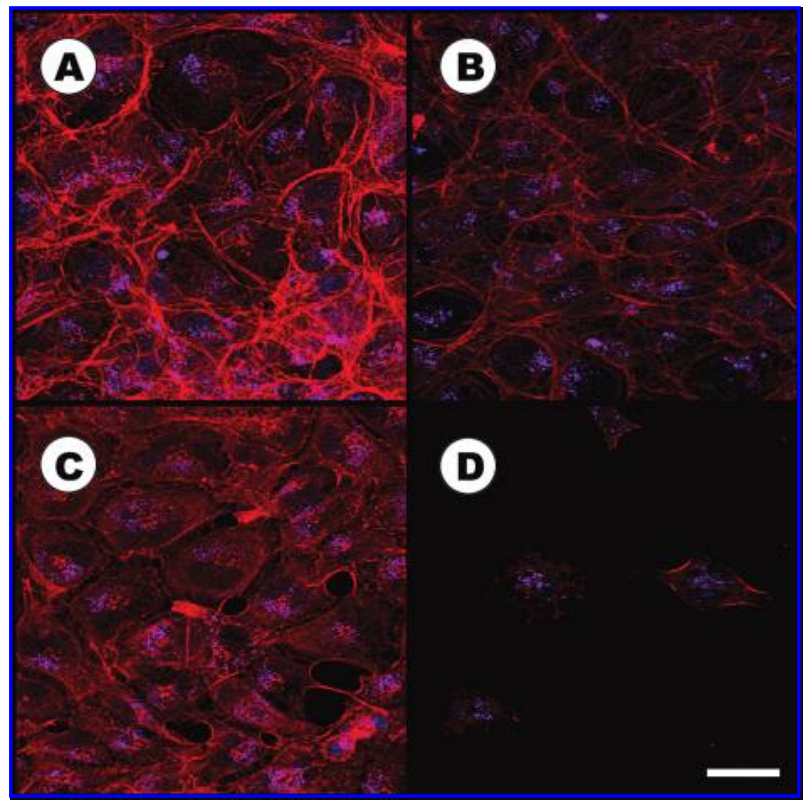

FIG. 5. Cells from a human lens epithelial cell line (SRA $04 / 01)$ were treated for $24 \mathrm{~h}$ with increasing concentrations of tamoxifen (A, $\mu M$; $\mathbf{B}, 5 \mu M$; $\mathbf{C}, 25 \mu M$; and $\mathbf{D}, 50 \mu M)$. Factin cytoskeleton was stained with rhodamine phalloidin (red), and nuclei were stained with TOTO-3 dye (blue). Qualitative presentation of actin cytoskeleton using confocal microscopy $(40 \times$ objective, $1.25 \mathrm{NA})$ shows a rapid drop in F-actin fluorescence at concentrations as low as $5 \mu M$. At $25 \mu M$ tamoxifen is starting to become cytotoxic, and actin fiber aggregation is observed. Finally, at $50 \mu M$ drug there is a total loss of microfilaments. 
tor (ADF)/cofilin, ${ }^{36}$ intracellular bacterial pathogens such as Listeria and lamellipodial and filopodial protrusion, ${ }^{24}$ and proteins that link integrins to the actin cytoskeleton, including talin, vinculin, $\alpha$-actinin, and filamin. ${ }^{39}$ Arguably, one of the most important cellular associations of the actin cytoskeleton involves the Rho family of small GTPases. 6,18,23 Rho GTPases regulate several actinbased cellular processes, including cell adhesion, cytokinesis, and contraction. ${ }^{19}$ By inducing coordinated changes in the organization of the actin cytoskeleton, Rho GTPases serve as molecular switches, activating biological responses ranging from morphogenesis to cell cycle guidance. ${ }^{6}$ Rho also plays a role in signaling pathways leading to the formation of actin stress fibers and focal adhesions - for example, in fibroblasts stimulated by lysophosphatidic acid (LPA). In 1997 Amano et al. ${ }^{18}$ showed that microinjection into serum-starved Swiss 3T3 cells of the catalytic domain (CAT) of Rho-kinase, a target protein activated by Rho, induced the formation of actin stress fibers and focal adhesions. Microinjection of three of the other four domains of Rho-kinase inhibited the LPA-induced formation of stress fibers and focal adhesions. Because injection of the CAT of Rho-kinase slightly increased the intensity of phalloidin staining, the authors conclude that Rho-kinase may marginally induce actin polymerization. Randomly arranged F-actin was observed in cells stimulated by LPA in the presence of the protein kinase inhibitor staurosporine, but not in cells injected with CAT in the presence of staurosporine.

Functional aberrations involving regulatory proteins such as Rho GTPases have been associated with various pathological conditions, as can be seen in several ophthalmologic studies centered on the lens. ${ }^{4} \mathrm{~A}$ transparent organ containing many proteins, the lens is responsible for focusing light on the retina, a process that requires the lens to vary its refractive power via accommodationi.e., by changing its shape. During growth of the lens, lens epithelial cells divide, lengthen, and differentiate into fiber cells. Differentiation remains an ongoing process throughout life, characterized by the synthesis and accumulation of cystallins, soluble proteins found in abundance in the lens. The accumulation of two crystallins in particular- $\alpha \mathrm{A}$ - and $\alpha \mathrm{B}$-crystallin-has been associated with several neoplastic and neurological disorders. ${ }^{4}$ In the lens, both proteins are known to undergo changes via phosphorylation and bind such cytoskeletal proteins as actin.

Despite gains made in recent decades in understanding lens biology, much remains unclear about the molecular processes regulating cytoskeletal organization, differentiation of fiber cells, and the abundance of crystallins in the lens - particularly as they relate to lens dysfunction and the formation of cataracts. In the early 1990s, analysis of Rho, newly identified as a member of the Ras superfamily of small GTPases, showed that its activation led to actin stress fiber formation-introducing yet another piece of the molecular puzzle. ${ }^{6}$ In a study published in 1997 using GTP blot overlay assays, Rao et $a l .{ }^{4}$ were the first to detect and characterize in the lens members of the family of GTP-binding proteins - small GTPases in the range of $20-30 \mathrm{kDa}$. At the same time, the authors identified what they concluded to be a new class of GTP-binding proteins: three distinct proteins ranging from 33 to $45 \mathrm{kDa}$. Because these three GTPbinding proteins were exclusively associated with the cortex and nucleus (i.e., fiber cells) and were not found in heart or brain tissue, the investigators believe they may be distinct from small GTPases $(20-30 \mathrm{kDa})$ as well as from heterotrimeric GTPases. Taken together, the detection of two types of GTP-binding proteins in the lens pointed to their involvement in such important cellular regulatory functions as growth, differentiation, and cytoskeleton organization. Furthermore, the likelihood that small GTPases play a significant role in these functions in the lens supports observations by Rao et al. ${ }^{4}$ and others linking these regulatory proteins to cataract formation via both genetic mutation and drug toxicity.

Through their role in lens cell differentiation - established in 1964 by the first description of microtubules in lengthening lens fibers - cytoskeletal proteins have been directly implicated in the formation of cataracts. ${ }^{5} \mathrm{Al}-$ though cytoskeletal proteins such as actin make up less than $5 \%$ of the proteins found in the lens, they are thought to serve perform critical functions related to lens transparency. In transparent lens cells, spatial fluctuations in the refraction index are small compared to the wavelength of visible light, and the cytoskeletal proteins are uniformly distributed. When the proteins form an insoluble mass large enough to approximate the dimensions of the wavelength of light, the index of refraction is enlarged, increasing the scattering of light, and dense opacification occurs. Among the biochemical changes that have been reported in human cataracts is the loss of cytoskeletal proteins, including actin. In a study of cataracts induced by selenite injection in rats, Matsushima et al. ${ }^{5}$ found that the formation of cataracts due to opacification and aggregation of insoluble proteins was associated with a rapid decrease in $\beta 1$-crystallin and several cytoskeletal proteins, including actin. The investigators also found that, by administering pantethine, a known anti-cataract agent, loss of cytoskeletal proteins could be inhibited along with opacification, and cataracts never developed. In fact, actin concentration in the lens nucleus increased in the rats treated with pantethine, while noncrystallin proteins with molecular masses of 42, 55/57, and 235 $\mathrm{kDa}$-labeled with antibodies against actin, tubulin/vimentin, and spectrin, respectively - were degraded during the earliest phases of cataract formation in rats injected with selenite. Finding that the loss of noncrystallin proteins may play an important role in the early phases 
of selenite cataract formation points to the importance of the vimentin-actin-tubulin component in establishing a transparent cell structure during lens differentiation in the cortex. Because crystallins were observed in the selenite cataract undergoing the process of partial proteolysis and insolubilization similar to what they undergo from birth to 4 months of age in the normal lens nucleus, the changes in protein composition during normal differentiation may require the same interactions between cytoskeleton and crystallins that they observed in the selenite cataracts. Loss of cytoskeletal proteins was associated with an abnormal variation in phase-separation temperature by day 3 following selenite injection and with membrane damage and loss of fiber structure by day 2 to 3 , indicating that a properly organized cytoskeleton may be necessary for normal development and maintenance of transparent lens structure. ${ }^{5}$

More recent research has associated actin cytoskeleton with such eye problems as retinal detachments and corneal disease as well as cataracts - in some cases, in further association with drug toxicity. Earlier studies had established that pharmacologically induced deterioration of cytoplasmic microfilaments results in submembrane alterations and then weakening of cellular adhesions in such cell types as those of the human trabecular meshwork. ${ }^{40}$ In a study in which HTM cells were treated with the actin-disrupting agent latrunculin-A (LAT-A) known to increase ocular outflow and reduce intraocular pressure, Cai et al. ${ }^{40}$ found that the drug induced marked but reversible rounding of the HTM cells as well as rapid but reversible loss of actin filaments and disruption of cellular adhesions. The results lead the authors to the view that interactions involving HTM cells and the underlying extracellular matrix may represent a target for pharmacological control of intraocular pressure. Because LATA exerts a facility-increasing control effect that is reversible in monkeys within $3 \mathrm{~h}$ after the drug is stopped, LAT-A may function by inducing a physiological modulation of a basic control mechanism instead of causing irreversible toxicity. Clinical applicability of LAT-A may be possible by targeting the drug specifically to the cells of the conventional outflow pathway. To achieve optimal outflow facility enhancement without increasing the risk of irreversible changes in the pathway or related tissues, the authors called for more research on the assembly and disassembly of actin and actin-associated adhesion proteins of HTM cells treated with LAT-A and other agents. Cai et al. ${ }^{40}$ concluded that, because the high concentrations of LAT-A in small volumes typically used in topical drug protocols and clinical therapy can potentially harm the cornea, use of lower concentrations in large volumes should be explored. Three years later, members of this same research group reported results demonstrating the mitigating effects of LAT-A on alterations to actin organization and other changes induced by dexametha- sone. ${ }^{10}$ Changes induced in HTM cells caused by glutocorticosteroids such as dexamethasone are known to include actin cytoskeletal reorganization, which, when combined with other effects of the drug, may lead to glaucoma. ${ }^{41,42}$ The ability of LAT-A to inhibit the effects of dexamethasone on the actin cytoskeleton in cultured HTM cells at a dose below the threshold for increasing outflow facility in monkeys suggests that LAT-A at low doses may be useful in treating glaucoma.

Other studies published in the past 3-4 years have linked the actin cytoskeleton to other forms of corneal disease, the world's most common form of bilateral blindness, and to retinal disease. ${ }^{1,9}$ The presence of Rho-associated protein kinase in rabbit cornea was linked to delayed epithelial cell migration - a cause of numerous disorders of the corneal epithelium - through the effects of several agents, including LPA, a Rho activator; exoenzyme C3, a Rho inhibitor; and cytochalasin B, an inhibitor of actin filament assembly. ${ }^{1}$ Two years later, phenotypes in the mouse mutants corneal disease-1 (corn 1) and corneal disease- ${ }^{2 \mathrm{~J}}\left(\operatorname{corn} 1^{2 \mathrm{~J}}\right)$ were shown to be caused by mutations within the destrin gene, more commonly referred to as ADF. ${ }^{9}$ Destrin, which is a member of the ADF/cofilin family of regulatory proteins responsible for enhancing the turnover of actin in vivo, is normally highly expressed in the corneal epithelium. Based on their observations that the two corn 1 mutations were deficient in destrin and increased the content of F-actin in corneal epithelial cells, Ikeda et al. ${ }^{9}$ concluded that the proper regulation of actin dynamics is necessary for normal maintenance of the corneal epithelium and suggested a new pathway through which aberrant actin cytoskeleton leads to epithelial proliferation. Similarly, cellular and cytoskeletal alterations observed in explant cultures of porcine neural retina detached from the retinal pigmented epithelium resembled degenerative changes seen following induced retinal detachments. ${ }^{8}$ The association, involving such alterations as the frequent, thick bundling of F-actin seen across the width of the retina, suggests that the cytoskeleton of individual cell types may play a key role in retinal damage.

The role of Rho and Rac GTPases, particularly their induced activation and inhibition in human lens epithelial cells, serves as a further link between the actin cytoskeleton and drug toxicity. ${ }^{43}$ As already discussed, earlier studies have suggested a role for the small GTPases in lens growth and development and in the maintenance of lens function, through such molecular mechanisms as membrane localization in various signal transduction pathways. For such mechanisms to occur, the small GTPases must undergo a process known as post-translational isoprenylation involving mevalonic acid and its derivatives, geranylgeranyl and farnesyl isoprenoids. Inhibition of the production of mevalonate and its isoprenoid derivatives, thus impairing small GTPase function, can be 
caused by 3-hydroxy-3-methylglutaryl coenzyme A (HMG-CoA) reductase inhibitors used to reduce cholesterol. Such HMG-CoA reductase inhibitors as lovastatin, simvastatin, and related compounds have been shown to cause cataracts in experimental models, and a mutation in mevalonate kinase has been associated with cataractogenesis in humans. ${ }^{44,45}$ In previous studies, Rao et al. ${ }^{4}$ found that the inhibition of small GTPase isoprenylation in lens epithelial cells treated with lovastatin led to significant changes in cell shape and reduced epithelial proliferation. Suggesting that such changes potentially activate cell death pathways as well as alteration to the cytoskeleton, the authors hypothesized that lovastatin-induced cataractogenesis may stem, at least in part, from impaired Rho and Rac GTPase function. In a study published in 2001, members of the same research group observed dramatic changes in human and porcine lens epithelial cells - marked by a "profound" loss of such components as actins stress fibers and focal adhesionsfollowing treatment with lovastatin. ${ }^{2}$ The authors also detected a resultant increase in nonisoprenylated Rho and Rac GTPases. By demonstrating that inhibition of GTPase isoprenylation by lovastatin leads to cellular loss and disruption of actin cytoskeletal organization, the results suggest that impairment of Rho and Rac GTPase function is, in the investigators' view, "most likely responsible" for lovastatin-induced changes in lens epithelial cells. In a 2003 study showing that pretreatment with such GTPase-inhibiting agents as lovastatin negated growth-factor stimulation of actin stress fiber formation in human lens epithelial cells, Maddala et al. ${ }^{3}$ found that the actin cytoskeleton "constitutes one of the critical down stream targets of growth factor activity in lens epithelial cells." They concluded that the ability of growth factors to activate actinomyosin cytoskeletal reorganization as well as Rho and Rac GTPases may be a critical factor in the proliferation, migration, elongation, and survival of lens epithelial cells.

Interestingly, the association of HMG-CoA reductase inhibitors such as lovastatin and related compounds with cataractogenesis may be due to a mechanism that has also been identified as a possible contributing factor to the anticancer activity of another commonly prescribed agent linked to cataracts, tamoxifen. ${ }^{43}$ The risk of cataracts has been shown to increase when cholesterol biosynthesis in the ocular lens is disrupted, such as by pharmacologic intervention. ${ }^{46}$ Although statins - which, as a class, are the most prescribed drugs in the United States-have not been linked directly to cataractogenesis in humans, inhibition of HMG-CoA reductase has been shown to increase the risk of cataracts in humans, tripling the risk in patients who received simvastatin concomitantly with two or more courses of erythromycin. ${ }^{47} \mathrm{~A}$ new generation of hypocholesterolemic drugs known as oxidosqualene cyclase inhibitors offers less secondary increase of
HMG-CoA reductase, among other pharmacologic advantages; however, these drugs may be more cataractogenic than statins. One oxidosqualene cyclase inhibitor, U18666A (developed by Pharmacia-Upjohn), has been associated with irreversible lens damage within several weeks after initiation of treatment, effects that could contribute to the formation of cataracts. ${ }^{43}$ In a study designed to test the hypothesis that cataracts caused by U18666A may be linked to direct perturbation of lens membrane structure and function, Cenedella et al $^{43}$ assessed alterations to membrane order or fluidity by measuring changes in fluorescent probe anisotropy and through the use of small-angle x-ray diffraction. Based on observations made using these methods, the investigators found that the presence of U18666A in animal lens lipid models increased membrane structural order and, through the drug's intercalation into the cell membranes, resulted in a broad condensing effect on membrane structure. Exposure to U18666A also induced apoptosis, which may have been another result of the intercalation that took place. In considering several hypotheses about the mechanism of the drug's toxicity, the authors cited evidence suggesting that the resulting cataracts cannot be explained solely by the ability of U18666A to restrict the level of cholesterol and that the toxic effects on the lens cell are due, instead, to an independent factor. A more likely explanation, in their view, is that direct intercalation of U18666A into the membrane bilayer, by leading to changes in the membrane structure, contributes to cataractogenesis. The anticancer effect of tamoxifenwhich, like U18666A, is a liphophilic-amphipathicethoxy tertiary amine - may be partially derived from the agent's ability to perturb membrane structure. ${ }^{48}$ It is perhaps not surprising, then, that treatment with tamoxifen is associated with an increased risk of cataracts. ${ }^{49}$

For the purposes of the study we undertook involving actin, several factors suggested that tamoxifen was an appropriate choice to demonstrate the effects of drug toxicity on the cytoskeleton. A nonsteroidal, oral anti-estrogen, tamoxifen has been widely used to treat advanced breast cancer and as postoperative therapy in patients with early-stage disease. ${ }^{26}$ Based largely on the findings of the National Surgical Adjuvant Breast and Bowel Project's Breast Cancer Prevention Trial (P-1) showing a reduced incidence of breast cancer among high-risk women receiving the drug, tamoxifen was approved by the U.S. Food and Drug Administration for preventive use, further expanding the large population of women using the agent. $^{64}$

Although tamoxifen has been shown to be generally well tolerated for a hormonal agent, its use has been associated with certain well-documented adverse effects. The earliest reports of ocular toxicity were made in 1978, and these were case reports of retinopathy in women being treated with very high doses for metastatic breast can- 
cer. ${ }^{50}$ Since these early reports, regular treatment with low-dose tamoxifen (20-40 mg/day) has been associated with bilateral optic neuritis, ${ }^{51-53}$ corneal deposits, $, 54,55$ retinal changes, ${ }^{56}$ glaucoma and macular degeneration, ${ }^{26}$ crystalline retinopathy, ${ }^{57}$ optic neuropathy, ${ }^{58}$ visual acuity abnormalities, macular edema, corneal opacities, and lens changes, among other forms of ocular toxicity. ${ }^{59} \mathrm{In}$ vestigators conducting the Breast Cancer Prevention Trial found that patients receiving tamoxifen had an increased risk of cataracts. ${ }^{26}$ The incidence of toxic reactions to tamoxifen has been estimated to be between $0.9 \%$ and $12 \%$, with the incidence of retinopathy in particular estimated at approximately $5 \%{ }^{60}$ Colley and Elston ${ }^{58}$ reported that this latter form of toxicity has been reversible in the majority of cases and suggested that prompt cessation of therapy may lead to visual recovery even in the case of toxic optic neuropathy. However, in a case report on tamoxifen maculopathy, ${ }^{60}$ it was concluded that halting tamoxifen therapy usually stops the progression of ocular disease but does not necessarily result in visual recovery. Based on the results of a follow-up study of women who participated in a trial assessing the effect of hormone replacement therapy on breast cancer risk suggested that 5 or more years of tamoxifen therapy increases risk of cataracts, ${ }^{61}$ the investigators recommend determining baseline visual function before initiating therapy, with regular eye exams afterward. They stopped short of suggesting cessation of therapy for those patients who do develop cataracts.

Taken together, the results of these studies suggest that tamoxifen ocular toxicity, if arrested early enough, can often be reversible. However, the mechanism for such effects, particularly with respect to the retina, remains unclear. As suggested above, there is a suggested association between axonal degeneration and the formation of crystalline retinal deposits in patients receiving tamoxifen. ${ }^{50}$ Alternatively, rats treated subchronically with high doses of tamoxifen were found to have a generalized lipidosis, leading investigators to hypothesize that the agent itself, by binding with polar lipids, inhibits lipid catabolism and promotes the buildup of drug-lipid complexes in lysosomes. ${ }^{62}$ With respect to in vitro tamoxifen toxicity in the human retinal pigmented epithelium, the activity of lysosomal enzymes in such cells is inhibited by tamoxifen, just as we have seen with the phagocytosis of rod outer segments in retinal pigmented epithelial cell cultures. ${ }^{14,63}$ Changes in membrane lipids may also provide an explanation of the underlying mechanism; although to our knowledge, there are no published reports of the effect of tamoxifen toxicity on the actin cytoskeleton. The reports regarding the phagocytosis of rod outer segments in the retinal pigmented epithelium suggest that toxic effects on actin would be demonstrable. The implication that many of these effects may be reversible also points up the importance of the relative cytotoxicity of the tamoxifen concentrations being used.

In our study we showed that cytoskeletal changes resulted from concentrations of tamoxifen ranging from, at the low end, 1-5 $\mu M$. These concentrations are among the lowest seen in trials involving tamoxifen and suggest that changes in actin cytoskeleton may serve as a more sensitive marker of retinal and lens toxicity than currently available assays. In several studies published since 2001, concentrations of tamoxifen have been shown to be toxic between $7.2 \pm 1.1 \mu M$ and $20.6 \mu M$ (50\% effective concentration) for human retinal pigmented epithelial cells and as low as $3.6 \pm 1.1 \mu M(50 \%$ effective concentration) for pig retinal pigmented epithelial cells, depending on the duration of exposure. ${ }^{14,31,32,48}$ Comparable results have also been obtained when researchers measured values using $\mu \mathrm{mol} / \mathrm{L}$ or measured $50 \%$ inhibitory concentration values. ${ }^{14,48}$ In a study of the effects of different concentrations of tamoxifen and two other agents on the phagocytosis of rod outer segments by the retinal pigmented epithelium, Mannerström et al. ${ }^{14}$ measured the phagocytized rod outer segments fluorometrically and assessed drug toxicity with the use of the WST-1 test. While tamoxifen reduced phagocytic activity in human as well as pig cells via both measures, the inhibition was induced at levels of tamoxifen below what the WST-1 test indicated was cytotoxic, suggesting that phagocytic activity was a more sensitive marker phenomenon to the test compounds than was the basic cytotoxicity. The WST-1 test offers the advantage of yielding water-soluble cleavage products, reducing the number of steps needed before measurements can be made. However, because the homeostatic regulatory functions of the retinal pigmented epithelium are critical to the retina as a whole, these functions should be measured along with cytotoxicity. The retinal pigmented epithelium is an interesting in vitro model for the evaluation of retinal toxicity; however, in addition to the WST-1 test, more specific tests and markers based on the homeostatic functions of the retinal pigmented epithelium are needed.

The results of our present study suggest that changes in the actin cytoskeleton may be one such specific marker. This assertion is made with several caveats. First, there is the issue of reproducibility. The results of this study, particularly with respect to the low cytotoxic concentrations of tamoxifen, need to be validated by further testing by other laboratories as well as our research group. In addition, the usefulness of actin as a biomarker would be further validated if similar results could be obtained using drug compounds besides tamoxifen. These two approaches represent the future line of research in our laboratory.

A second caveat has to do with the limitations of our study. Although the study consisted of a series of relatively simple, straightforward experiments using known 
in vivo toxicants producing what we found to be clear results, the lack of consensus within the field on the underlying mechanism(s) by which tamoxifen affects the lens and retina makes it difficult to definitely conclude why the drugs are causing changes in the actin cytoskeleton at such low concentrations. Is tamoxifen cytotoxicity related to decreased enzymatic activity, or is it due to the alternative explanation of simple reduced phagocytic activity? The latter may be attributable to such drug/membrane interactions as protein $\mathrm{C}$ kinase activation induced by tamoxifen and occurring via the phospholipase pathway. ${ }^{48}$ Further research is needed to identify the precise role of the actin cytoskeleton in any of these processes.

Finally, there is the question of the utility of an actin cytoskeletal biomarker in drug screening for ocular toxicity due to its ubiquitous nature in cell biology. Yet, the opportunity exists to build on this review to develop screening assays that are more sensitive and specific toward their intended purpose. For ocular toxicity, preselecting compounds based on their ability to alter the actin cytoskeleton in cells may be an appropriate mechanism to prioritize development candidates. Such screening of drugs used for chronic treatment of debilitating or lifethreatening diseases can be controversial, as reported with respect to hydroxychloroquine and chloroquine retinopathy due to overdosing, where the debate revolved around the ability of ophthalmologic tests to detect early retinopathy. ${ }^{27}$ Although dosing options - including the decision to continue or discontinue a drug exhibiting ocular toxicity-will belong to the physician treating the original disease, informed consent of patients experiencing adverse ocular effects can be improved with the use of more precise ophthalmologic tests and markers.

The development of drug safety screens is untraditional in the sense that most screens are out to qualify a drug target or screen compounds to a target on the basis of efficacy. Toxicology, on the other hand, is a sensitive measure of the exaggeration of pharmacology, but really lacks specificity. The present study has provided a potential application for a simple observation, and others are encouraged to apply their molecular screens to answer questions in toxicology. The present extensive discussion is provided so that groups currently investigating actin biology may broaden the use of their molecular efforts to try to ascribe a mechanism to the observations described here. This may be the link needed to increase the specificity of the ocular toxicity observation. In this manner, researchers may not only be able to report that cataracts were observed, but that they were produced by a perturbation of a specific biochemical pathway. With this knowledge, it is possible for medicinal chemists to alter a particular test compound to not stimulate this pathway. This is just one way that a compound could be de-risked, which is a term commonly used when obtaining a balance between compound efficacy and safety. Our findings suggest that the actin cytoskeleton may have a role in such a clinical advance.

\section{Acknowledgments}

The authors wish to express their appreciation to Daniel Baltrukonis, Chris Somps, and Amy Jakowski for their continued support in this area of investigation.

\section{References}

1. Nakamura M, Nagano T, Chikama T, Nishida T: Role of the small GTP-binding protein rho in epithelial cell migration in the rabbit cornea. Invest Ophthalmol Vis Sci 2001;42:941-947.

2. Maddala RL, Reddy VN, Rao PV: Lovastatin-induced cytoskeletal reorganization in lens epithelial cells: role of Rho GTPases. Invest Ophthalmol Vis Sci 2001;42:2610-2615.

3. Maddala R, Reddy VN, Epstein DL, Rao V: Growth factor induced activation of Rho and Rac GTPases and actin cytoskeletal reorganization in human lens epithelial cells. Mol Vis 2003;9:329-336.

4. Rao PV, Zigler JS Jr, Garland D: Analysis of small GTPbinding proteins of the lens by GTP overlay assay reveals the presence of unique GTP-binding proteins associated with fiber cells. Exp Eye Res 1997;64:219-227.

5. Matsushima H, David LL, Hiraoka T, Clark JI: Loss of cytoskeletal proteins and lens cell opacification in the selenite cataract model. Exp Eye Res 1997;64:387-395.

6. Hall A: Rho GTPases and the actin cytoskeleton. Science 1998;279:509-514.

7. McGough A: F-actin-binding proteins. Curr Opin Struct Biol 1998;8:166-176.

8. Winkler J, Hagelstein S, Rohde M, Laqua H: Cellular and cytoskeletal dynamics within organ cultures of porcine neuroretina. Exp Eye Res 2002;74:777-788.

9. Ikeda S, Cunningham LA, Boggess D, Hawes N, Hobson CD, Sundberg JP, et al:: Aberrant actin cytoskeleton leads to accelerated proliferation of corneal epithelial cells in mice deficient for destrin (actin depolymerizing factor). Hum Mol Genet 2003;12:1029-1037.

10. Liu X, Wu Z, Sheibani N, Brandt CR, Polansky JR, Kaufman PL: Low dose latrunculin-A inhibits dexamethasoneinduced changes in the actin cytoskeleton and alters extracellular matrix protein expression in cultured human trabecular meshwork cells. Exp Eye Res 2003;77:181-188.

11. Young RW, Bok D: Participation of the retinal pigment epithelium in the rod outer segment renewal process. $\underline{\mathrm{J} \mathrm{Cell}}$ Biol 1969;42:392-403.

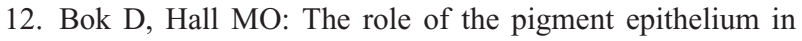
the etiology of inherited retinal dystrophy in the rat. $\underline{\mathrm{JCell}}$ Biol 1971;49:664-682.

13. Edwards RB, Szamier RB: Defective phagocytosis of isolated rod outer segments by RCS rat retinal pigment epithelium in culture. Science 1977;197:1001-1003.

14. Mannerström M, Maenpaa $H$, Toimela T, Salminen L, Tahti H: The phagocytosis of rod outer segments is inhibited by selected drugs in retinal pigment epithelial cell cultures. Pharmacol Toxicol 2001;88:27-33. 
15. Fisher SK, Steinberg RH: Origin and organization of pigment epithelial apical projections to cones in cat retina. $\underline{J}$ Comp Neurol 1982;206:131-145.

16. Greenberg S: Signal transduction of phagocytosis. Trends Cell Biol 1995;5:93-99.

17. Kwiatkowska K, Sobota A: Signaling pathways in phagocytosis. Bioessays 1999;21:422-431.

18. Amano M, Chihara K, Kimura K, Fukata Y, Nakamura N, Matsuura Y, et al.: Formation of actin stress fibers and focal adhesions enhanced by Rho-kinase. Science 1997;275: 1308-1311.

19. Narumiya S, Ishizaki $\mathrm{T}$, Watanabe $\mathrm{N}$ : Rho effectors and reorganization of actin cytoskeleton. FEBS Lett 1997;410: $68-72$.

20. Matozaki T, Nakanishi H, Takai Y: Small G-protein networks: their crosstalk and signal cascades. Cell Signal 2000;12:515-524.

21. Li W, Chong H, Guan KL: Function of the Rho family GTPases in Ras-stimulated Raf activation. J Biol Chem 2001; 276:34728-34737.

22. Christerson LB, Gallagher E, Vanderbilt CA, Whitehurst AW, Wells C, Kazempour R, et al:: p115 Rho GTPase activating protein interacts with MEKK1. J Cell Physiol 2002;192:200-208

23. Small JV, Rottner K, Kaverina I: Functional design in the actin cytoskeleton. Curr Opin Cell Biol 1999;11:54-60.

24. Borisy GG, Svitkina TM: Actin machinery: pushing the envelope. Curr Opin Cell Biol 2000;12:104-112.

25. Mejico LJ, Bergloeff J, Miller NR: New therapies with potential neuro-ophthalmologic toxicity. Curr Opin Ophthalmol 2000;11:389-394.

26. Paganini-Hill A, Clark LJ: Eye problems in breast cancer patients treated with tamoxifen. Breast Cancer Res Treat 2000;60:167-172.

27. Browning DJ: Hydroxychloroquine and chloroquine retinopathy: screening for drug toxicity. Am J Ophthalmol 2002;133:649-656.

28. Kennedy CJ, Rakoczy PE, Robertson TA, Papadimitriou JM, Constable IJ: Kinetic studies on phagocytosis and lysosomal digestion of rod outer segments by human retinal pigment epithelial cells in vitro. Exp Cell Res 1994;210: 209-214.

29. Smith-Thomas LC, Richardson PS, Rennie IG, Palmer I, Boulton M, Sheridan C, et al:: Influence of pigment content, intracellular calcium and cyclic AMP on the ability of human retinal pigment epithelial cells to contract collagen gels. Curr Eye Res 2000;21:518-529.

30. Dufour EM, Nandrot E, Marchant D, Van Den BL, Gadin $\mathrm{S}$, Issilame $\mathrm{M}$, et al.: Identification of novel genes and altered signaling pathways in the retinal pigment epithelium during the Royal College of Surgeons rat retinal degeneration. Neurobiol Dis 2003;14:166-180.

31. Maenpaa H, Saransaari P, Tahti H: Kinetics of inhibition of glutamate uptake by antioestrogens. Pharmacol Toxicol 2003;93:174-179.

32. Engelke M, Bojarski P, Bloss R, Diehl H: Tamoxifen perturbs lipid bilayer order and permeability: comparison of DSC, fluorescence anisotropy, laurdan generalized polarization and carboxyfluorescein leakage studies. Biophys Chem 2001;90:157-173.

33. Ibaraki N, Chen SC, Lin LR, Okamoto H, Pipas JM, Reddy VN: Human lens epithelial cell line. Exp Eye Res 1998; 67:577-585

34. Higley S, Way M: Actin and cell pathogenesis. Curr Opin Cell Biol 1997;9:62-69.
35. Walczak CE: Microtubule dynamics and tubulin interacting proteins. Curr Opin Cell Biol 2000;12:52-56.

36. Goode BL, Drubin DG, Barnes G: Functional cooperation between the microtubule and actin cytoskeletons. Curr Opin Cell Biol 2000;12:63-71.

37. Rogers SL, Gelfand VI: Membrane trafficking, organelle transport, and the cytoskeleton. Curr Opin Cell Biol 2000; 12:57-62.

38. Mullins RD: How WASP-family proteins and the Arp2/3 complex convert intracellular signals into cytoskeletal structures. Curr Opin Cell Biol 2000;12:91-96.

39. Critchley DR: Focal adhesions - the cytoskeletal connection. Curr Opin Cell Biol 2000;12:133-139.

40. Cai S, Liu X, Glasser A, Volberg T, Filla M, Geiger B, et al.: Effect of latrunculin-A on morphology and actin-associated adhesions of cultured human trabecular meshwork cells. Mol Vis 2000;6:132-143.

41. Clark AF, Lane D, Wilson K, Miggans ST, McCartney MD: Inhibition of dexamethasone-induced cytoskeletal changes in cultured human trabecular meshwork cells by tetrahydrocortisol. Invest Ophthalmol Vis Sci 1996;37:805-813.

42. Clark AF, Wilson K, McCartney MD, Miggans ST, Kunkle M, Howe W: Glucocorticoid-induced formation of cross-linked actin networks in cultured human trabecular meshwork cells. Invest Ophthalmol Vis Sci 1994;35: 281-294.

43. Cenedella RJ, Jacob R, Borchman D, Tang D, Neely AR, Samadi A, et al.: Direct perturbation of lens membrane structure may contribute to cataracts caused by U18666A,

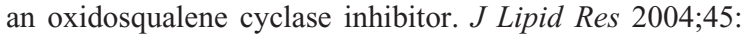
1232-1241.

44. Cenedella RJ: Inhibitors of cholesterol synthesis and cataracts. JAMA 1987;257:1602.

45. Hoffmann G, Gibson KM, Brandt IK, Bader PI, Wappner RS, Sweetman L: Mevalonic aciduria - an inborn error of cholesterol and nonsterol isoprene biosynthesis. $\mathrm{N} \mathrm{Engl} \mathrm{J}$ Med 1986;314:1610-1614.

46. Cenedella RJ: Cholesterol and cataracts. Surv Ophthalmol 1996;40:320-337.

47. Schlienger RG, Haefeli WE, Jick H, Meier CR: Risk of cataract in patients treated with statins. Arch Intern Med 2001;161:2021-2026.

48. Engelke M, Tykhonova S, Zorn-Kruppa M, Diehl H: Tamoxifen induces changes in the lipid composition of the retinal pigment epithelium cell line D407. Pharmacol Toxicol 2002;91:13-21.

49. Gorin MB, Day R, Costantino JP, Fisher B, Redmond CK, Wickerham L, et al.: Long-term tamoxifen citrate use and potential ocular toxicity. Am J Ophthalmol 1998;125: 493-501.

50. Kaiser-Kupfer MI, Lippman ME: Tamoxifen retinopathy. Cancer Treat Rep 1978;62:315-320.

51. Pugesgaard T, Von Eyben FE: Bilateral optic neuritis evolved during tamoxifen treatment. Cancer 1986;58: 383-386.

52. Ashford AR, Donev I, Tiwari RP, Garrett TJ: Reversible ocular toxicity related to tamoxifen therapy. $\underline{\text { Cancer }} 1988$; 61:33-35.

53. Noureddin BN, Seoud M, Bashshur Z, Salem Z, Shamseddin A, Khalil A: Ocular toxicity in low-dose tamoxifen: a prospective study. Eye 1999;13:729-733.

54. Pavlidis NA, Petris C, Briassoulis E, Klouvas G, Psilas C, Rempapis J, et al.: Clear evidence that long-term, low-dose tamoxifen treatment can induce ocular toxicity. A prospective study of 63 patients. Cancer 1992;69:2961-2964. 
55. Heier JS, Dragoo RA, Enzenauer RW, Waterhouse WJ: Screening for ocular toxicity in asymptomatic patients treated with tamoxifen. Am J Ophthalmol 1994;117: $772-775$

56. Tang R, Shields J, Schiffman J, Li H, Locher D, Hampton $\mathrm{J}$, et al.: Retinal changes associated with tamoxifen treatment for breast cancer. Eye 1997;11:295-297.

57. Lazzaroni F, Scorolli L, Pizzoleo CF, Savini G, De Nigris A, Giosa F, et al.: Tamoxifen retinopathy: does it really exist? Graefes Arch Clin Exp Ophthalmol 1998;236: 669-673.

58. Colley SM, Elston JS: Tamoxifen optic neuropathy. Clin Exp Ophthalmol 2004;32:105-106.

59. Nayfield SG, Gorin MB: Tamoxifen-associated eye disease. A review. J Clin Oncol 1996;14:1018-1026.

60. Alwitry A, Gardner I: Tamoxifen maculopathy. Arch Ophthalmol 2002;120:1402.

61. Ross RK, Paganini-Hill A, Wan PC, Pike MC: Effect of hormone replacement therapy on breast cancer risk: estrogen versus estrogen plus progestin. $\underline{\text { J Natl Cancer Institute }}$ 2000;92:328-332.

62. Lullmann H, Lullmann-Rauch R: Tamoxifen-induced generalized lipidosis in rats subchronically treated with high doses. Toxicol Appl Pharmacol 1981;61:138-146.
63. Toimela T, Salminen L, Tahti H: Effects of tamoxifen, toremifene and chloroquine on the lysosomal enzymes in cultured retinal pigment epithelial cells. Pharmacol Toxicol 1998;83:246-251.

64. Fisher B, Costantino JP, Wickerham DL, Redmond CK, Kavanah M, Cronin WM, Vogel V, Robidoux A, Dimitrov N, Atkins J, Daly M, Wieand S, Tan-Chin E, Ford L, Wolmark N: Tamoxifen for prevention of breast cancer: report of the National Surgical Adjuvant Breast and Bowel Project P-1 Study. J Natl Cancer Inst 1998;90:1371-1388.

Address reprint requests to: Mark W. Tengowski, D.V.M., M.S., Ph.D. Pfizer Global Research \& Development Michigan Laboratories Ann Arbor Campus 2800 Plymouth Road MS 16-1A/6 Ann Arbor, MI 48105

E-mail: mark.w.tengowski@pfizer.com 

This article has been cited by:

1. T P Barros, W K Alderton, H M Reynolds, A G Roach, S Berghmans. 2008. Zebrafish: an emerging technology for in vivo pharmacological assessment to identify potential safety liabilities in early drug discovery. British Journal of Pharmacology 154:7, 1400-1413. [CrossRef] 